\title{
Aspectos prosódicos temporais da leitura de escolares com dislexia do desenvolvimento
}

\author{
Temporal prosodic aspects of reading in students with \\ developmental dyslexia
}

\author{
Luciana Mendonça Alves', César Augusto da Conceição Reis², Ângela Maria Vieira Pinheiro², \\ Simone Aparecida Capellini ${ }^{4}$
}

\begin{abstract}
RESUMO
Objetivo: Analisar, do ponto de vista fonético, por meio de medidas acústicas e perceptivas, os aspectos prosódicos temporais presentes na leitura em voz alta de escolares com e sem dislexia, a fim de identificar diferenças de desempenho entre os dois tipos de leitores que possam apontar para características peculiares da dislexia. Métodos: Gravação da leitura de um texto por 40 escolares (entre nove e 14 anos, cursando da $3^{\text {a à }} 5^{\text {a }}$ série), sendo 10 disléxicos (grupo clínico) e 30 escolares sem queixas de alterações de aprendizagem (grupo não-clínico). Os dados foram analisados perceptivamente e acusticamente, utilizando-se o programa WinPitch. As seguintes medidas foram realizadas: duração e localização das pausas, tempo total de elocução, taxa de elocução, tempo de articulação e taxa de articulação. Resultados: Em comparação com o grupo não-clínico, o grupo clínico apresentou maior número de pausas e pausas mais longas; os valores obtidos para as taxas de elocução e de articulação indicaram respectivamente menor velocidade de leitura e uma lentidão na produção de cada gesto articulatório. Conclusão: As dificuldades identificadas no processamento da leitura pelas crianças com dislexia dificultam a organização prosódica na leitura de um texto.
\end{abstract}

Descritores: Dislexia; Leitura; Fonética; Aprendizagem; Acústica

\section{INTRODUÇÃO}

A dislexia tem sido amplamente investigada há mais de um século sob a ótica das mais variadas áreas de conhecimento. Questões neurológicas, genéticas, psicológicas, pedagógicas, linguísticas têm sido levantadas e descobertas e constatações importantes têm sido feitas nos últimos anos. No entanto, quando a dislexia é considerada sob a perspectiva linguística, nota-se uma carência de estudos na área da prosódia. Este aspecto linguístico dos disléxicos tem sido raramente tratado na literatura e, quando o é, principalmente nas pesquisas brasi-

Trabalho realizado na Universidade Estadual de Campinas - UNICAMP Campinas (SP), Brasil.

(1) Doutora, Professora do Centro Universitário Metodista Izabela Hendrix - Belo Horizonte (MG), Brasil.

(2) Pós-doutor, Professor da Universidade Federal de Minas Gerais - UFMG - Belo Horizonte (MG), Brasil.

(3) Pós-doutora, Professora Titular da Universidade Federal de Minas Gerais - UFMG - Belo Horizonte (MG), Brasil.

(4) Pós-doutora, Professora Assistente do Departamento de Fonoaudiologia e do Programa de Pós-Graduação em Educação da Faculdade de Filosofia e Ciências da Universidade Estadual Paulista "Júlio de Mesquita Filho" UNESP - Marília (SP), Brasil.

Endereço para correspondência: Luciana Mendonça Alves. R. Itinga, 85, Serrano, Belo Horizonte - MG, CEP: 32110-020. E-mail: lumendonca@terra.com.br

Recebido em: 26/6/2008; Aceito em: 11/8/2008 leiras, são feitas apenas observações superficiais. Na tentativa de preencher essa lacuna, o presente trabalho procura entender a leitura da criança disléxica com referência a aspectos linguísticos, em particular os prosódicos temporais. Caso uma relação entre esses aspectos e a dislexia seja encontrada, a descrição do perfil das características prosódicas temporais associadas à dislexia poderá ser utilizada como mais um recurso a ser empregado nos procedimentos de avaliação e de intervenção dessa condição.

De acordo com o Manual Diagnóstico e Estatístico de Transtornos Mentais ${ }^{(1)}$ (DSM-IV, 2002), a dislexia do desenvolvimento ou transtorno da leitura é caracterizada por um rendimento de leitura, inferior ao esperado para a idade cronológica, a inteligência medida e a escolaridade do indivíduo.

É importante destacar que essa condição deve ser diferenciada das variações na realização acadêmica e das dificuldades escolares decorrentes de déficits sensoriais, cognitivos, emocionais e de fatores educacionais, sócio-culturais e econômicos adversos.

Tomando-se por base os processos de leitura, a dificuldade específica na aquisição da linguagem escrita pelos disléxicos se expressa por falhas no processamento lexical ou fonológico (ou em ambos) que podem, por sua vez, ter sua origem nos processos sensoriais, ocasionando assim subtipos diferentes de dislexia, a depender do processo afetado. A dislexia fono- 
lógica, a mais comum, caracteriza-se por uma disfunção no processo fonológico, que se revela por deficiências no processo de decodificação fonológica (a associação entre grafema e fonema), necessário para a leitura de palavras pouco familiares. $\mathrm{Na}$ dislexia visual/lexical, o transtorno é no processamento visual das palavras. Na dislexia mista, os leitores apresentam alterações dos dois tipos (fonológica e visual) ${ }^{(2-4)}$.

Grande parte dos disléxicos apresenta déficits primários no processamento fonológico que são evidenciados por dificuldades nas tarefas que medem esse processo, tais como: tarefas que envolvem repetição de palavras e de não-palavras, retenção de material verbal na memória de trabalho, nomeação rápida de palavras e de figuras e manipulações metalinguísticas tais como a adição, subtração e transposição de fonemas ${ }^{(5)}$. Ou seja, para os autores referenciados, o problema central da dislexia é mais bem descrito como sendo um déficit fonológico, oriundo de alterações no processamento fonológico em tempo real.

Inúmeras são as teorias que tentam explicar a etiologia da dislexia. O que sabemos hoje é que existem fatores hereditários e fatores relacionados ao comportamento social, à lateralização cerebral e a uma disfunção cerebral, relacionados ao quadro de dislexia ${ }^{(6-8)}$.

Embora estudos tradicionais apontem que o hemisfério direito do cérebro (o hemisfério não-verbal) é o responsável pelo processamento da prosódia, enquanto que o hemisfério esquerdo processa a linguagem verbal, pesquisas recentes revelam que o processamento prosódico envolve múltiplas áreas em ambos os hemisférios, havendo áreas em comum com o processamento fonológico e as frequentemente apontadas como disfuncionais em indivíduos disléxicos ${ }^{(9)}$. É possível, pois, que haja uma provável relação entre as alterações prosódicas e a leitura dos escolares disléxicos.

O termo prosódia pode ser usado tanto para se referir aos sistemas cognitivos abstratos quanto aos parâmetros físicos nos quais esses sistemas abstratos são mapeados. No nível fonológico abstrato, pode-se distinguir a prosódia lexical (tom, acento e quantidade) da prosódia não-lexical (entonação). $\mathrm{O}$ termo entonação refere-se especificamente às características fonéticas dos enunciados, à forma pela qual as características prosódicas abstratas não-lexicais de um enunciado são relacionadas aos parâmetros acústicos ${ }^{(10)}$. A implementação fonética dessas categorias prosódicas é medida por meio dos parâmetros acústicos: frequência fundamental, intensidade e tempo.

A entonação pode ser então entendida como uma categoria prosódica abstrata e representa a elaboração linguística da melodia, estando no nível fonético ${ }^{(11-12)}$, adotando múltiplas funções em um discurso, em vários planos de análise. Estas funções são assumidas contextualmente e de forma socialmente configurada. Caracteriza-se como uma possibilidade para se conhecer melhor a leitura das crianças disléxicas, o que há sob o discurso delas, mesmo sem que elas saibam que estejam expressando seus sentimentos, atitudes e intenções.

Por intermédio do parâmetro tempo, estuda-se a organização temporal do enunciado, medindo-se a duração de segmentos e sílabas ${ }^{(13)}$. Na literatura, que considera a prosódia na leitura dos disléxicos, encontramos apenas referência ao parâmetro duração que não só é inadequadamente referido como "velocidade de leitura", como também impreciso, já que a velocidade de leitura é tomada como lenta, adequada ou rápida, ao invés de basear-se nas taxas de elocução e articulação. Estas taxas serão medidas e descritas neste trabalho, propondo-se, assim, um adequado tratamento dos aspectos prosódicos temporais da leitura das amostras estudadas.

Propomos, desta forma, analisar, do ponto de vista fonético, por meio de análise acústica e perceptiva, os aspectos prosódicos temporais presentes na leitura em voz alta das crianças disléxicas, comparando-os à leitura em voz alta de seus pares - crianças do grupo não-clínico, sem queixas de alterações de linguagem ou de aprendizagem - a fim de verificar quais são as características peculiares da dislexia.

\section{MÉTODOS}

O projeto que deu origem ao presente trabalho foi submetido ao Comitê de Ética da Faculdade de Ciências Médicas da Universidade Estadual de Campinas (UNICAMP), tendo sido aprovado sob o protocolo $n^{\circ}$ 029/2005.

A fim de averiguar o efeito da condição dislexia nos aspectos prosódicos temporais da leitura dos escolares com esse quadro, dois grupos foram estudados. O primeiro, com 10 membros, que receberam diagnóstico de dislexia fonológica - grupo clínico (GC) - e o outro, com 30 membros, sem esse diagnóstico e sem queixas de outras alterações de linguagem ou aprendizagem - grupo não-clínico (GNC). Ambos os grupos foram compostos por escolares entre nove e 14 anos, cursando da $3^{\mathrm{a}}$ à $5^{\mathrm{a}}$ série em escolas da rede pública de ensino, na cidade de Campinas (SP) e região.

A coleta de dados do GC foi realizada no Ambulatório de Neuro-Dificuldades de Aprendizagem do Hospital das Clínicas da Faculdade de Ciências Médicas da Universidade Estadual de Campinas (UNICAMP). Os escolares desse grupo foram avaliados por uma equipe interdisciplinar, constando de avaliação neurológica, neuropsicológica, pedagógica e fonoaudiológica com o diagnóstico confirmado de dislexia do desenvolvimento caracterizado por: presença de alteração quanto ao equilíbrio estático, à coordenação apendicular, persistência motora, equilíbrio dinâmico, coordenação tronco-membro e sensibilidade no exame neurológico evolutivo; discrepância entre coeficiente intelectual verbal e execução na avaliação psicológica, alteração quanto à memória, leitura e escrita na bateria neuropsicológica, alterações fonêmicas, silábicas, rima e aliteração em provas de consciência fonológica, nível de leitura alfabético, velocidade de leitura oral abaixo do esperado para idade e escolaridade, transtorno fonológico evidenciado na avaliação fonológica, na leitura oral de textos e na leitura oral de palavras isoladas e na escrita sob ditado de palavras e pseudo-palavras e na redação temática, compreensão parcial do texto lido.

No grupo de escolares com o diagnóstico confirmado de dislexia do desenvolvimento (GC), metade da amostra foi submetida a um programa de remediação fonológica, ou seja, um programa de tratamento constando de 20 sessões baseadas no desenvolvimento das habilidades de consciência fonológica ${ }^{(14)}$. Assim, o grupo clínico (GC) foi dividido em dois subgrupos: um grupo de cinco crianças que não passou por nenhum tipo de tratamento - GC I, e um grupo de cinco crianças que passou 
pelo programa de remediação fonológica descrito na referência mencionada - GC II.

Já os escolares do grupo não-clínico (GNC) são oriundos de duas escolas da rede estadual de ensino. Eles foram selecionados pelos professores por meio de questionário específico ${ }^{(15)}$. É comum que exista uma discrepância entre a avaliação da professora e a avaliação cognitiva de leitura conduzida posteriormente aos alunos pré-selecionados ${ }^{(16)}$. Para diminuir o risco desta discrepância e aumentar a confiabilidade de nossos dados, aplicou-se aos professores regentes das classes uma escala $^{(15)}$ que permitiu a classificação dos alunos, por critérios objetivos, em três categorias: a) alunos que lêem bem; b) alunos que lêem mais ou menos; c) alunos que lêem mal. A partir desta lista classificatória foram escolhidos os escolares classificados como bons leitores, de modo a compor o número amostral de 30 escolares sem queixas de alterações de linguagem oral e escrita. Utilizou-se, neste estudo, um grupo-controle composto por três vezes mais indivíduos do que o grupo-experimental, com o intuito de aumentar a validade interna dos achados.

A coleta de dados, com gravação da leitura oral dos escolares do GNC foi realizada na própria escola, individualmente, em uma sala à parte, em horário não prejudicial à aprendizagem do conteúdo curricular. Já a gravação dos escolares disléxicos (GC) foi realizada no Ambulatório de Neuro-dificuldades de Aprendizagem do Hospital das Clínicas da Faculdade de Ciências Médicas da UNICAMP, também de forma individual.

Em primeiro lugar, a pesquisadora deixou claro aos escolares pesquisados que não se tratava de um procedimento de avaliação, para que eles ficassem um pouco mais à vontade. Foi apresentado o texto "O tatu encabulado"(17) e solicitou-se, individualmente, aos escolares de cada grupo que o lessem em voz alta. Primeiramente, era sugerida uma leitura silenciosa do texto, antes de proceder à leitura em voz alta. Assim que o estudante sinalizasse que estava pronto, começava-se a gravação da leitura em voz alta.

O equipamento utilizado para a gravação foi um microfone de cabeça, da marca LeSon ${ }^{\circ}$ HD-75, unidirecional (cardióide), posicionado em uma distância de cerca de cinco centímetros dos lábios do escolar e conectado a um computador do tipo portátil da marca HP Pavilion® ZE 2210. A gravação foi feita por meio do programa Sound Forge 6.0 (com entrada de 16 bits de quantização e frequência de amostragem de $22050 \mathrm{~Hz}$ ) e analisada acusticamente pelo programa WinPitch Pro.

A leitura de cada escolar foi transcrita ortograficamente. Cada enunciado foi editado e analisado separadamente. Para a análise dos aspectos temporais, observamos as seguintes medidas:

- duração em milissegundos (ms) e localização das pausas: foram medidas as pausas (tanto as pausas silenciosas momentos de silêncio marcados pela ausência de sinal acústico - quanto as preenchidas por interjeições e outras locuções, demonstrativas de hesitação contida) dentro das frases e entre as frases, por meio de análise perceptiva e acústica instrumental;

- número de sílabas emitidas: foi utilizada a divisão silábica fonética, por meio de percepção auditiva;

- tempo total de elocução: refere-se ao tempo total de duração da leitura do texto, em segundos (s);
- taxa de elocução: esta medida é obtida dividindo-se o número total de sílabas emitidas pela duração completa do texto, ou seja, seu tempo de elocução, em sílabas/segundo ( $\mathrm{sil} / \mathrm{s})$;

- tempo de articulação: este é avaliado subtraindo-se da duração total do texto a duração total das pausas, em segundos $^{(13)}$;

- taxa de articulação: esta taxa é obtida dividindo-se o número total de sílabas emitidas pelo tempo de articulação, em sílabas/segundo ${ }^{(13)}$.

Observe-se que as quatro últimas medidas são relacionadas à velocidade de fala.

O tratamento estatístico dos dados consistiu do cálculo das médias, desvios padrão e os coeficientes de variação para cada variável estudada, em cada grupo. Em seguida, foi conduzida uma comparação entre os grupos e, para cada comparação, foi investigada a diferença entre as médias por meio de análises de variância (Teste F de Fischer-Snedecor). A série foi tomada como fator de bloco, de forma a termos o efeito da série minimizado. O nível de significância utilizado foi o de $5 \%(\mathrm{p}<0,05)$. Ou seja, sempre que o valor de $\mathrm{p}$ foi menor do que 0,05 , a diferença entre as médias foi considerada estatisticamente significante.

\section{RESULTADOS}

As análises estatísticas, realizadas em cada grupo (GC como um todo e separado em GC I e GC II - e GNC), compararam os valores encontrados nas variáveis acústicas prosódicas temporais para a leitura de texto efetuada por cada grupo e verificaram as diferenças obtidas entre os grupos. Conforme esperado, os estudantes do GNC apresentaram desempenho melhor e mais homogêneo na leitura do texto do que o GC. Leram com velocidade adequada, evidenciando reconhecimento lexical - tipo de leitura em que a ortografia e a pronúncia das palavras são acessadas da memória lexical. Em contraste, a leitura do GC (tanto como um todo quanto para cada subgrupo), foi caracterizada por uma grande heterogeneidade de desempenho, variando de leitura do tipo silabada, com erros de decodificação a leitura com poucos erros e fluente. Também a "velocidade de leitura" foi muito variada.

A Tabela 1 mostra as médias e desvios padrão (entre parênteses), obtidos para as variáveis prosódicas temporais investigadas para todos os grupos estudados. O padrão de resultados encontrado caracterizou-se, como esperado, por um melhor desempenho do GNC, suido pelo GC II e finalmente pelo GC I. Essa configuração de resultados foi expressa, por um lado, por médias maiores (acompanhadas de maiores desvios padrão) para o GC I e, gradativamente, menores para o GC II e para o GNC (acompanhadas de menores desvios padrão) para as variáveis: tempo total de elocução, tempo de articulação, número de sílabas, número de pausas, e duração das pausas. Por outro lado, observam-se médias menores para os grupos GC II e I, respectivamente, e maiores para o GNC para as variáveis: taxa de elocução e taxa de articulação, sendo que a última variável diferiu-se da primeira por não apresentar um padrão crescente ou decrescente de médias, tomando como base a ordem GC I, GC II e GNC. Essa variável apresentou 
médias mais altas para o GNC e mais baixas para o GC II, porém com um desvio padrão bem maior no GC I, o que indica uma maior variação de desempenho para esse grupo.

Em conjunto, esse panorama geral dos resultados nos dá os primeiros indícios de perfis bem definidos pelos grupos pesquisados com melhor desempenho pelo GNC e, assim, sucessivamente.

A Tabela 2 apresenta os valores relativos ao teste estatístico utilizado para verificar a diferença das médias entre os grupos pesquisados. O teste utilizado foi o teste $\mathrm{F}$, cujo valor é apresentado à esquerda, e o valor de $\mathrm{p}$ foi apresentado entre parêntesis, à direita. Sempre que este valor for menor do que 0,05 , a diferença entre as médias dos grupos comparados pode ser considerada estatisticamente significante.

Todos os valores obtidos de $\mathrm{p}$, ao se comparar o GNC com o GC e seus subgrupos, foram inferiores a 0,05 , ou seja, a diferença entre as médias foi estatisticamente significante para todas as variáveis pesquisadas. A seguir, apresentaremos os resultados de cada variável.

\section{Tempo total de elocução}

No que se refere à média do tempo total de elocução (tempo total despendido com a leitura), verificamos que a diferença entre o GNC e o GC foi significante, tanto em relação ao GC I quanto em relação ao GC II. É notável que o tempo total de elocução para o GNC, foi substancialmente menor (média de 46,94 s) do que para os subgrupos clínicos que apresentaram tempos muito longos de leitura (médias de 205,10 e 119,5 s, respectivamente).

\section{Tempo de articulação}

O tempo de articulação, cuja medida foi obtida pela subtração da duração total do texto pela duração das pausas, também se apresentou significantemente maior no GC, com o valor médio de 92,80 s para o GC I e de 90,6 s para o GC II (Tabela 1). Há também, neste caso, uma grande diferença entre estes valores para o encontrado no GNC, de 35,07 s.

\section{Taxa de elocução}

Lembramos que a mensuração da taxa de elocução consta do número de sílabas emitidas dividido pelo tempo de elocução, obtendo-se assim a taxa de sílabas lidas por segundo (sil/s). Essa medida tem sido referida por alguns autores como velocidade de fala, porém salientamos que o termo "taxa de elocução" nos fornece uma dimensão mais precisa dessa variável. A taxa média encontrada foi de 1,68 sil/s para o GC I e 1,758 sil/s para o GC II (Tabela 1), sendo que a diferença entre esses dois grupos não se mostrou estatisticamente significante (Tabela 2). Já os escolares do GNC atingiram uma taxa média de 3,69 sil/s. Os resultados do GNC em contraste com os resultados do GC e de seus subgrupos mostram diferenças estatisticamente significantes (Tabela 2) para todas as comparações, o que indica que o grupo de escolares com dislexia

Tabela 1. Aspectos prosódicos temporais

\begin{tabular}{|c|c|c|c|c|}
\hline \multirow[t]{2}{*}{ Variável } & \multicolumn{4}{|c|}{ Médias ( \pm Desvio padrão) } \\
\hline & GC (geral) & GC I & GC II & GNC \\
\hline Tempo total de elocução (s) & $162,3( \pm 135,90)$ & $205,10( \pm 182,20)$ & $119,5( \pm 61,10)$ & $46,94( \pm 9,48)$ \\
\hline Tempo de articulação (s) & $91,70( \pm 66,20)$ & $92,80( \pm 86,90)$ & $90,6( \pm 48,20)$ & $35,07( \pm 5,37)$ \\
\hline Taxa de elocução (sil/s) & $1,72( \pm 0,85)$ & $1,68( \pm 0,86)$ & $1,758( \pm 0,93)$ & $3,69( \pm 0,56)$ \\
\hline Taxa de articulação (sil/s) & $3,11( \pm 1,79)$ & $3,59( \pm 2,41)$ & $2,62( \pm 0,91)$ & $4,86( \pm 0,61)$ \\
\hline Número de sílabas & $213,6( \pm 62,50)$ & $219,80( \pm 80,70)$ & $207,4( \pm 46,80)$ & $167,67( \pm 6,61)$ \\
\hline Número de pausas & $97,80( \pm 64,70)$ & $104,60( \pm 67,70)$ & $91,0( \pm 68,70)$ & $22,83( \pm 5,08)$ \\
\hline Duração de pausas (ms) & $86,00( \pm 87,70)$ & $101,40( \pm 103,30)$ & $70,7( \pm 77,80)$ & $11,53( \pm 3,72)$ \\
\hline
\end{tabular}

Legenda: GC = grupo clínico; GNC = grupo não-clínico; s = segundos; sil/s = sílabas por segundo

Tabela 2. Análise estatística dos aspectos prosódicos temporais

\begin{tabular}{lcccc}
\hline Variável & \multicolumn{4}{c}{ Teste F (Valor de p) } \\
\cline { 2 - 5 } & GNC - GC & GNC - GC I & GNC - GC II & GC I - GC II \\
\hline Tempo total de elocução (s) & $12,52(0,00)$ & $23,23(0,00)$ & $36,41(0,00)$ & $0,05(0,84)$ \\
Tempo de articulação (s) & $8,71(0,00)$ & $13,25(0,00)$ & $85,91(0,00)$ & $0,11(0,76)$ \\
Taxa de elocução (sil/s) & $49,69(0,00)$ & $62,37(0,00)$ & $56,30(0,00)$ & $0,94(0,40)$ \\
Taxa de articulação (sil/s) & $8,83(0,03)$ & $05,37(0,03)$ & $54,90(0,00)$ & $1,64(0,29)$ \\
Número de sílabas & $7,34(0,00)$ & $12,29(0,00)$ & $38,67(0,00)$ & $0,04(0,86)$ \\
Número de pausas & $26,69(0,00)$ & $45,71(0,00)$ & $101,8(0,00)$ & $0,31(0,62)$ \\
Duração de pausas (ms) & $12,84(0,00)$ & $23,56(0,00)$ & $87,77(0,00)$ & $0,03(0,87)$ \\
\hline
\end{tabular}

Legenda: GC = grupo clínico; GNC = grupo não-clínico; s = segundos; sil/s = sílabas por segundo 
apresenta uma velocidade mais lenta para ler um texto do que os seus pares sem queixas de alterações na linguagem.

\section{Taxa de articulação}

Uma menor taxa de articulação também foi evidenciada no GC. Esta taxa, obtida pela divisão do número de sílabas emitidas pelo tempo de articulação, indicou a velocidade de produção do gesto articulatório para a leitura, cujos valores também são apresentados em sil/s.

No GNC, o seu valor médio foi de 4,86 sil/s (Tabela 1) e tanto se comparando ao GC I, com média de 3,59 sil/s, quanto ao GC II, com 2,62 sil/s, esta taxa foi significantemente maior (Tabela 2), o que indica uma lentidão na produção de cada gesto articulatório para a leitura por escolares disléxicos. Assim como as demais variáveis, esta diferença também não se mostrou significante ao conduzirmos comparações entre os grupos GC I e GC II.

\section{Número de sílabas}

O número de sílabas produzidas durante leitura em voz alta foi significantemente menor para o GNC com, em média, 167,67 sílabas, sendo que, no GC I, foram contadas, em média, 219,80 sílabas e, no GC II, 207,4 sílabas (Tabela 1). A comparação do GC I com o GC II, como nas demais variáveis, não resultou em diferença estatisticamente significante.

\section{Número de pausas}

Com relação ao número de pausas, foi observado que os escolares do GNC se utilizaram de um número bem menor, com média de 22,83 pausas (Tabela 1 ), de modo a apresentar significância estatística (Tabela 2) quando comparado à média obtida para o GC I, de 104,60 pausas, e para o GC II, de 91 pausas. Além do uso preferencial das pausas silenciosas, que ocorreu nos três grupos repetições, sendo as repetições de sílabas a mais frequente.

\section{Duração de pausas}

As médias para as variáveis número e duração das pausas foram bastante diferentes entre o GC e o GNC (Tabela 1), com o último grupo apresentando médias significantemente menores do que a média de todos os GC como nos mostra a Tabela 2. Essa tabela também nos mostra que para as duas medidas consideradas a diferença entre os dois subgrupos clínicos não foi significante.

\section{Localização das pausas}

Na utilização das pausas, houve também diferenças significantes entre os grupos pesquisados. Podemos observar, pela Tabela 3, que enquanto os escolares do GNC apresentaram um número significantemente menor de pausas $(22,83 \mathrm{em}$ média, ocorrendo predominantemente entre os grupos tonais, locais esperados para a sua ocorrência), os escolares do GC utilizaram mais pausas (97,5 em média). Essas, ao contrário do GNC, foram realizadas predominantemente dentro dos grupos tonais (55 em média) e com frequência também alta, dentro de palavras (23,5 em média), sendo a frequência nessas posições significantemente maior para o GC, com uma ocorrência mínima para o GNC.

Verificamos que a média de ocorrência de pausas entre os grupos tonais é equivalente para ambos os grupos (em torno de 14 pausas), o que demonstra que também os indivíduos disléxicos fizeram algumas pausas nos locais adequados em sua leitura. Acrescidas a estas, houve utilização de pausas dentro dos grupos tonais, em um número que chega a ser mais do que o triplo das utilizadas em seus locais convencionais, além das pausas dentro de palavras.

Os resultados das comparações entre o GNC com os dois subgrupos clínicos (GC I e II), (Tabela 4), sugerem o mesmo padrão obtido anteriormente. Mostram que no que se refere às pausas entre os grupos tonais, apesar de sua maior frequência na leitura dos escolares do GNC, não houve diferença significante entre esse grupo e os dois subgrupos clínicos e nem entre esses subgrupos entre si (Tabela 5). Já ao considerarmos as duas outras posições em que as pausas foram identificadas, observamos que os grupos clínicos exibiram um maior número de pausas dentro dos grupos tonais, seguido de pausas dentro das palavras, com o grupo GC I apresentando médias nos dois locais $(63,4$ e 26,8, respectivamente) superiores às médias obtidas pelo grupo GC I (46,6 e 20,2, respectivamente). No entanto, como nos mostra a Tabela 5 , as diferenças entre os dois grupos clínicos, no que se refere a essas posições, não

Tabela 3. Análise estatística para a localização das pausas durante a leitura comparação entre GNC e GC

\begin{tabular}{lccccc}
\hline Localização das pausas & Grupo & Média & Dp & Teste F & Valor de $p$ \\
\hline Entre grupos tonais & GNC & 14,9 & 2,28 & 3,85 & 0,15 \\
\hline Dentro de grupos tonais & GC & 14,6 & 4,06 & 17,06 & 0,00 \\
\hline Dentro de palavras & GNC & 7,37 & 50,4 & 2301,00 \\
& GC & 55 & 0,63 & 27,3 & 18,23 \\
\hline Total de pausas & GNC & 0,57 & 5,00 & 0,00 \\
\end{tabular}

Legenda: GC = grupo clínico; GNC = grupo não-clínico; Dp = desvio padrão 
Tabela 4. Média e desvio padrão da localização das pausas durante a leitura comparação entre todos os grupos

\begin{tabular}{llcc}
\hline Localização das pausas & Grupo & Média & Dp \\
\hline Entre grupos tonais & GC I & 13 & 1,58 \\
& GC II & 16,2 & 3,49 \\
& GNC & 14,9 & 2,28 \\
\hline Dentro de grupos tonais & GC I & 63,4 & 40,8 \\
& GC II & 46,6 & 42,8 \\
\hline Dentro de palavras & GNC & 7,37 & 4,80 \\
\hline Total de pausas & GC I & 26,8 & 25,8 \\
& GC II & 20,2 & 31,5 \\
& GNC & 0,57 & 0,63 \\
\hline & GC I & 104 & 67 \\
& GC II & 91 & 68,7 \\
\hline
\end{tabular}

Legenda: $\mathrm{GC}=$ grupo clínico; $\mathrm{GNC}=$ grupo não-clínico; $\mathrm{Dp}=$ desvio padrão

foram significantes, ao passo que o foram ao compararmos o GNC com o GC I e o GNC com o GC II.

\section{DISCUSSÃO}

A primeira observação sobre a leitura de texto por nossas amostras foi a grande heterogeneidade de desempenho pelos escolares disléxicos em comparação com as suas contrapartes. Esse resultado não é surpreendente já que os achados da literatura $^{(16)}$ também apontam para uma grande variação na capacidade de leitura por crianças com dificuldade nessa habilidade. A comparação entre o GC I e II mostrou que a heterogeneidade identificada foi maior no GC I, o que nos leva a concluir que a intervenção terapêutica recebida pelo GC II teve, pelo menos, um efeito qualitativo (menor variância) no desempenho de leitura desse grupo, já que os dois subgrupos clínicos não se diferiram no que se refere às analises quantitativas.

De uma maneira geral, os resultados do presente estudo são condizentes com as descrições encontradas na literatura, tanto para os participantes do GNC, quanto aos do $\mathrm{GC}^{(1,18-20)}$. No tocante ao primeiro grupo, de acordo com nossas expectativas, todos os seus membros apresentaram leitura proficiente, com resultados para cada uma das variáveis testadas dentro do esperado e de acordo com a literatura. Por exemplo, em pesquisas conduzidas com amostras equivalentes de escolares sem comprometimentos de linguagem, as médias para tempo de articulação (36,62 e 40,36 s) $)^{(21-22)}$, de taxa de articulação $(4,82 \mathrm{sil} / \mathrm{s})^{(22)}$ e de duração de pausas $(9,13 \mathrm{~ms})^{(22)}$ foram bem próximas às médias por nós encontradas, confirmando, assim, a classificação dos membros do grupo sob consideração como bons leitores. Relembrando, essa classificação foi obtida por meio da escala respondida pelo professor ${ }^{(15)}$.

Os achados referentes ao maior tempo total de elocução obtido pelos escolares do GC são coerentes com as dificuldades encontradas por este grupo para a atividade de leitura. Estes apresentam pausas mais longas e mais frequentes, pois despendem um tempo maior com as tentativas de decodificação. Os valores obtidos não podem ser comparados a outras pesquisas realizadas com esta medida, pois é uma variável que depende, além do tempo individual de organização da fala no tempo, do tamanho do texto lido.

Os valores de tempo de articulação encontrados para o trabalho com escolares com transtorno de aprendizagem ${ }^{(21)}$ evidenciam valores menores $(62,71 \mathrm{~s})$ do que os obtidos na presente pesquisa, o que demonstra que os escolares com dislexia apresentam uma maior lentidão no tempo de produção da articulação para a leitura do que os escolares com transtorno de aprendizagem. Ou seja, os escolares com dislexia, mesmo desconsiderando-se as pausas utilizadas na leitura, demandaram um tempo bem maior na articulação dos segmentos da fala para a leitura.

Também os valores de taxa de elocução dos grupos GC I e GC II apresentaram-se similares aos encontrados em outra pesquisa $^{(21)}$ para escolares com transtorno de aprendizagem $1,41 \mathrm{sil} / \mathrm{s}$. Estes resultados que indicam uma leitura mais lenta nas crianças com dislexia em função das dificuldades de leitura deste grupo já eram esperados ${ }^{(23)}$.

Corroborando os resultados obtidos para a taxa de articulação, temos novamente referência a pesquisas similares com valores médios de $2,65 \mathrm{sil} / \mathrm{s}$ para escolares da mesma faixa etária da pesquisada com transtorno de aprendizagem ${ }^{(21)}$, o que indica que a velocidade de produção do gesto articulatório é semelhante entre escolares com dislexia e com transtorno de aprendizagem.

Esses valores reduzidos da taxa de articulação, evidenciados no GC, estão também condizentes com outra pesquisa ${ }^{(24)}$ que demonstra que a velocidade de produção de cada gesto articulatório é mais lenta em crianças com distúrbio específico de leitura.

Conforme já salientado, a taxa de articulação foi a única variável temporal que não sugeriu um padrão de resultados em que o GC II apresentou desempenho qualitativamente melhor do que o do GC I. Era de se esperar que os escolares que foram submetidos ao programa de reeducação em leitura apresentassem semelhantemente aos membros do GNC, maior velocidade de produção do gesto articulatório. Isso porque

Tabela 5. Análise estatística para a localização das pausas durante a leitura comparação entre todos os grupos

\begin{tabular}{|c|c|c|c|c|c|c|c|c|}
\hline & \multicolumn{2}{|c|}{ Entre grupos tonais } & \multicolumn{2}{|c|}{ Dentro de grupos tonais } & \multicolumn{2}{|c|}{ Dentro de palavras } & \multicolumn{2}{|c|}{ Total de pausas } \\
\hline & Teste F & Valor de $p$ & Teste F & Valor de $p$ & Teste F & Valor de $p$ & Teste F & Valor de $p$ \\
\hline GC I - GC II & 3,21 & 0,073 & 0,1 & 0,754 & 0,28 & 0,59 & 0,1 & 0,75 \\
\hline GNC - GC I & 2,18 & 0,094 & 11,91 & 0,001 & 14,37 & 0 & 11,59 & 0,001 \\
\hline GNC - GC II & 0,59 & 0,443 & 7,53 & 0,006 & 13,99 & 0 & 9,17 & 0,002 \\
\hline
\end{tabular}

Legenda: GC = grupo clínico; GNC = grupo não-clínico 
uma maior facilidade na habilidade de decodificação poderia permitir uma produção mais rápida da leitura oral. No entanto, uma possível explicação para esse achado é que a intervenção recebida tenha tido o efeito de tornar os escolares tratados mais conscientes de suas dificuldades na leitura, levando-os a uma produção mais lenta (cuidadosa) de cada gesto articulatório, na tentativa de uma decodificação mais precisa.

Quanto aos resultados obtidos para o número de sílabas, não podemos compará-los aos obtidos por outras pesquisas, pois os valores são diretamente relacionados ao tamanho do texto lido. O número menor de sílabas encontrado nos escolares do GNC deve-se a fatores como a maior proximidade da forma de produção da leitura com a fala espontânea (talvez uma leitura com maior "naturalidade"), aparecendo em situações como os fenômenos de sândi (que ocorrem nas junturas intervocabulares, transformando estas estruturas silábicas por situações, como queda de vogais e formação de ditongos). Por outro lado, os escolares disléxicos decodificam, em algumas partes do texto, cada sílaba lida e produzem às vezes repetições destas, enquanto processam os elementos seguintes a serem decodificados, o que causa um aumento no número de sílabas produzidas.

As pausas constituem-se em um recurso extremamente importante para a organização temporal do discurso e, por meio delas, pode-se verificar a organização discursiva em relação à atitude do falante (hesitação, colaboração, interação com o tema), ao gênero discursivo (narração, argumentação, descrição) e ao estilo do discurso (texto oral espontâneo ou leitura). As pausas não-silenciosas são raras em textos de leitura, uma vez que, nestes, não há necessidade de planejamento do discurso ${ }^{(25)}$. Tal fato pode ser observado em nosso corpus, em que as pausas silenciosas foram mais frequentes, tendo-se também observado repetições, especialmente as de sílabas. Normalmente, esse é um recurso utilizado como um tempo que o leitor pensa "ganhar" na tentativa de decodificar o próximo segmento da palavra. Assim, enquanto tenta decodificar a sílaba seguinte repete automaticamente, a anterior. Geralmente, isso acontece diante de palavras novas para as quais é necessária a utilização da rota fonológica.

O maior número de pausas observado no GC justifica-se pelas dificuldades verificadas no processamento do texto impresso, precisando haver um tempo entre a visualização do item a ser lido e sua decodificação.

Os achados referentes à duração das pausas também se mostram próximos aos obtidos pela pesquisa com escolares com diagnóstico de transtorno de aprendizagem $(68,9 \mathrm{~ms})^{(21)}$.

Portanto, da mesma forma que os escolares disléxicos necessitam de um maior número de pausas para processar o texto lido, conforme discutido anteriormente, eles também necessitam que estas pausas sejam mais longas do que as utilizadas pelos seus pares do GNC. Ou seja, eles demandam mais tempo no processamento das unidades do texto.

Devemos enfatizar que o pequeno tamanho do grupo de disléxicos estudado não nos permite fazer generalizações acerca do perfil dessa população. O número de participantes de amostras clínicas de condições que ocorrem em baixa frequência na população, como é o caso da dislexia, é um fator limitante para a pesquisa. A despeito disso, os achados do presente estudo permitem delinear algumas características associadas a um perfil que pode ser atribuído aos escolares disléxicos pesquisados com relação a aspectos prosódicos temporais, como a utilização excessiva das pausas, estando estas fora da sua localização habitual além da velocidade de leitura e articulação reduzidas.

O padrão de enunciados e pausas na leitura oral contribui para a medida da fluência na leitura. Dessa forma, a prosódia, por si só, não pode ser uma determinante da fluência na decodificação de palavras, mas pode ser vista como uma medida diagnóstica para a qualidade da decodificação de palavras e a compreensão da leitura ${ }^{(26)}$. É extremamente interessante esta visão das habilidades prosódicas como medida diagnóstica, o que vem reforçar a importância do presente trabalho, sendo que esta visão é compartilhada por outros autores ${ }^{(27-28)}$.

Outro alcance deste estudo foi obtido em relação aos resultados do programa de remediação das habilidades de consciência fonológica direcionado ao GC $\mathrm{II}^{(14)}$. A autora relata após as 20 sessões de desenvolvimento das habilidades metacognitivas, uma melhora no nível e velocidade de leitura, além de melhores habilidades de consciência fonológica em si, o que resultou em uma melhor decodificação. No entanto, nossas medidas objetivas dos aspectos prosódicos temporais não evidenciaram este comportamento de melhora na velocidade de leitura em relação ao grupo que não passou pelo processo de remediação. Para todas as variáveis prosódicas temporais medidas, os resultados não se apresentaram significantemente melhores do que os obtidos pelo GC I. Este achado ressalta a importância de se incluir no processo de avaliação da leitura as medidas aqui apresentadas, que não fazem parte dos procedimentos utilizados na prática clínica convencional.

Sabe-se que estamos ainda longe de esgotar o assunto e que mais estudos em áreas afins precisam ser desenvolvidos para se elucidar a questão da dislexia, as características da leitura desses escolares e seus atributos prosódicos. Entretanto, acredita-se que este trabalho fornece dados importantes para um melhor conhecimento de um assunto até então muito pouco explorado: a relação entre prosódia e leitura em indivíduos com dislexia.

\section{CONCLUSÃO}

As características dos aspectos prosódicos temporais pareceram, em grande parte, diretamente relacionadas às dificuldades de decodificação do texto. Os valores elevados de tempo de articulação, número e duração das pausas (que estiveram presentes em locais fora do esperado para a sua ocorrência, como dentro de palavras) e os valores baixos de taxa de elocução remetem-se a esta relação. No entanto, os valores inferiores de taxa de articulação encontrados para os disléxicos levam à constatação de que existe uma lentidão no gesto articulatório para a produção da leitura, o que pode não ser diretamente atribuído às dificuldades de decodificação, mas também consequência de todo o processo de dificuldades com este processamento em diversos níveis. Mas, para que se possa chegar a conclusões definitivas, dever-se-ia investigar a fala espontânea dos disléxicos, a fim de se excluir a possibilidade de ser a lentidão do gesto articulatório uma peculiaridade da fala desses indivíduos. 
Concluímos que o nosso estudo corroborou as nossas hipóteses, sobretudo, a de que as dificuldades que a criança disléxica apresenta no processamento de textos escritos parecem dificultar a organização prosódica na leitura de um texto. Não podemos deixar, no entanto, de apontar a necessidade de se prosseguir na busca de uma melhor compreensão da leitura dos disléxicos, abordando as lacunas deixadas por este estudo, em investigações complementares.

\section{AGRADECIMENTOS}

Nossos agradecimentos à Dra. Sylvia Ciasca, que prontamente disponibilizou o Ambulatório de Transtornos da Aprendizagem - DISAPRE para a nossa coleta de dados. Nosso muito obrigado também à Fga. MS. Cíntia Salgado pela grande cooperação para a realização deste trabalho.

\begin{abstract}
Purpose: To analyze the temporal prosodic aspect in loud reading of students with and without dyslexia from the phonetic point of view through acoustic and perceptual evaluation, in order to identify differences in performance between the two types of readers that may point to particular characteristics of dyslexia. Methods: Forty students from $3^{\text {rd }}$ to $5^{\text {th }}$ grades with ages ranging from nine to 14 years, ten dyslexic (clinical group) and 30 without any complaints of learning deficits (non-clinical group) were recorded during loud reading of a text. Data were perceptually and acoustically analyzed using the software WinPitch. The following measures were taken: pause duration and location, total speaking time, speaking rate, articulation time, and articulation rate. Results: In comparison to the non-clinical group, the clinical group had a higher number of and longer pauses; their speaking and articulation rates indicated, respectively, a lower reading speed and slowness in the production of each articulatory gesture. Conclusion: The characteristics identified in reading processing by dyslexic children make the prosodic organization in text reading difficult.
\end{abstract}

Keywords: Dyslexia; Reading; Velocity measurement; Learning; Acoustics

\section{REFERÊNCIAS}

1. American Psychiatric Asociation. Manual diagnóstico e estatístico de transtornos mentais. 4a ed. Porto Alegre: Artmédica; 2002.

2. Gombert JE. Implicit and explicit learning to read: implication as for subtypes of dyslexia. Curr Psychol Lett. 2003;10(1):98-102.

3. Boose ML, Valdois S. Patterns of developmental dyslexia according to a multi-trace memory model of reading. Curr Psychol Lett. 2003;10(1):6570.

4. Manis FR, Seidenberg MS, Doi LM, McBride-Chang C, Petersen A. On the bases of two subtypes of developmental [corrected] dyslexia. Cognition. 1996;58(2):157-95. Erratum in: Cognition. 1996;59(2):245.

5. Blomert L, Mittere H, Paffen C. In search of the auditory, phonetic, and/or phonological problems in dyslexia: context effects in speech perception. J Speech Lang Hear Res. 2004;47(5):1030-47.

6. Lozano A, Ramírez M, Ostrosky-Solís F. Neurobiología de la dilexia del desarrollo: uma revisión. Rev Neurol. 2003;36(11):1077-82.

7. Fisher SE, DeFries JC. Developmental dyslexia: genetic dissection of a complex cognitive trait. Nat Rev Neurosci. 2002;3(10):767-80.

8. Shaywitz SE, Shaywitz BA. Dyslexia (specific reading disability). Biol Psychiatry. 2005;57(11):1301-9. Review.

9. Riecker A, Wildgruber D, Dogil G, Grodd W, Ackermann H. Hemispheric lateralization effects of rhythm implementation during syllable repetitions: an fMRI study. Neuroimage. 2002;16(1):169-76

10. Hirst DJ. Form and function in the representation of speech prosody. Speech Commun. 2005;46(3-4):334-47.

11. Rossi M. A Principle-based model for predicting the prosody of speech. In: Sorin C. Levels in speech communication: relations and interactions. Amsterdam: Elsevier Science; 1995.

12. Hirst D, Di Cristo A, editors. Intonation systems: a survey of twenty languages. Cambridge, U.K.; New York, NY: Cambridge University Press; 1998.

13. Grosjean F, Collins M. Breathing, pausing and reading. Phonetica. 1979;36(2):98-114.

14. Salgado CA, Capellini SA. Programa de remediação fonológica em escolares com dislexia do desenvolvimento. PróFono.2008;20(1):31-6.
15. Pinheiro AMV, Costa AEB. Escala de Avaliação de Competência em Leitura pelo Professor [resumo]. In: VII Encontro Mineiro de Avaliação Psicológica - EMAP, 2005, Belo Horizonte; 2005. p. 27-27.

16. Pinheiro AMV. Heterogeneidade entre leitores julgados competentes pelas professoras. Psicol Reflex Crít. 2001;14(3):537-51.

17. Scliar-Cabral L. Guia prático de alfabetização: baseado em princípios do sistema alfabético do português do Brasil. São Paulo: Contexto; 2003.

18. Nation K, Norbury CF. Why reading comprehension fails: insights from developmental disorders. Top Lang Disord. 2005;25(1):5-20.

19. Barros AFF, Capellini SA. Avaliação fonológica, de leitura e escrita em crianças com distúrbio específico de leitura. J Bras Fonoaudiol. 2003;4(14):11-9.

20. Pinheiro AMV. Reading and spelling development in Brazilian Portuguese. Reading \& Writing. 1995;7(1):111-38.

21. Alves LM, Pinheiro A, Reis C, Capellini S, Reis C. Aspectos temporais e entonativos na leitura e compreensão de crianças com transtorno de aprendizagem. Rev Soc Bras Fonoaudiol. 2006;11(3):151-7.

22. Celeste LC, Coradi CA, Alves LM, Reis C. Organização temporal na leitura de crianças. In: IX Congresso Nacional e III Congresso Internacional de Fonética e Fonologia. Anais. Belo Horizonte: Universidade Federal de Minas Gerais; 2006.

23. Capellini SA, Cavalheiro LG. Avaliação do nível e da velocidade de leitura em escolares com e sem dificuldade na leitura. Temas Desenvolv. 2000;9(51):5-12.

24. Fawcett AJ, Nicolson RI. Children with dyslexia are slow to articulate a single speech gesture. Dyslexia. 2002;8(4):189-203.

25. Yacovenco LC. O fenômeno prosódico da pausa e a organização temporal do discurso. Bol Assoc Bras Ling (ABRALIN). 2001;26(1):720-3.

26. Breznitz Z. Fluency in reading: synchronization of processes. Mahwah: Lawrence Erlbaum Associates; 2006.

27. Kuhn MR, Stahl SA. Fluency: a review of developmental and remedial practices. J Educ Psychol. 2003;95(1):3-21.

28. Schwanenflugel PJ, Hamilton AM, Kuhn MR, Wisenbaker JM, Stahl SA. Becoming a fluent reader: reading skill and prosodic features in the oral reading of young readers. J Educ Psychol. 2004;96(1):119-29. 